Research Article

\title{
Identification of Core Gene Biomarkers in Patients with Diabetic Cardiomyopathy
}

\author{
Ning Li $\mathbb{D},{ }^{1}$ Haiming Wu, ${ }^{1}$ Rongxin Geng $\mathbb{D}^{2},{ }^{2}$ and Qizhu Tang $\mathbb{D}^{1}$ \\ ${ }^{1}$ Department of Cardiology, Renmin Hospital of Wuhan University, Cardiovascular Research Institute of Wuhan University, \\ Hubei Key Laboratory of Cardiology, Wuhan, China \\ ${ }^{2}$ Department of Neurosurgery, Renmin Hospital of Wuhan University, China
}

Correspondence should be addressed to Qizhu Tang; qztang@whu.edu.cn

Received 25 June 2018; Revised 30 August 2018; Accepted 6 September 2018; Published 19 December 2018

Guest Editor: Liam M. Heaney

Copyright (C) 2018 Ning Li et al. This is an open access article distributed under the Creative Commons Attribution License, which permits unrestricted use, distribution, and reproduction in any medium, provided the original work is properly cited.

Diabetic cardiomyopathy (DCM) is a disorder of the myocardium in diabetic patients, which is one of the critical complications of diabetes giving rise to an increased mortality. However, the underlying mechanisms of DCM remain incompletely understood presently. This study was designed to screen the potential molecules and pathways implicated with DCM. GSE26887 involving 5 control individuals and 7 DCM patients was selected from the GEO database to identify the differentially expressed genes (DEGs). DAVID was applied to perform gene ontology (GO) and the Kyoto Encyclopedia of Genes and Genomes (KEGG) analyses. A protein-protein interaction (PPI) network was also constructed to visualize the interactions among these DEGs. To further validate significant genes and pathways, quantitative real-time PCR (qPCR) and Western blot were performed. A total of 236 DEGs were captured, including 134 upregulated and 102 downregulated genes. GO, KEGG, and the PPI network disclosed that inflammation, immune disorders, metabolic disturbance, and mitochondrial dysfunction were significantly enriched in the development of DCM. Notably, IL6 was an upregulated hub gene with the highest connectivity degree, suggesting that it may interact with a great many molecules and pathways. Meanwhile, SOCS3 was also one of the top 15 hub genes in the PPI network. Herein, we detected the protein level of STAT3 and SOCS3 in a mouse model with DCM. Western blot results showed that the protein level of SOCS3 was significantly lower while phosphorylated-STAT3 (P-STAT3) was activated in mice with DCM. In vitro results also uncovered the similar alterations of SOCS3 and P-STAT3 in cardiomyocytes and cardiac fibroblasts induced by high glucose (HG). However, overexpression of SOCS3 could significantly reverse HG-induced cardiomyocyte hypertrophy and collagen synthesis of cardiac fibroblasts. Taken together, our analysis unveiled potential biomarkers and molecular mechanisms in DCM, which could be helpful to the diagnosis and treatment of DCM.

\section{Introduction}

Diabetic cardiomyopathy (DCM) is a common cardiac dysfunction which affects approximately $12 \%$ of diabetic patients, giving rise to overtly higher cardiovascular morbidity and mortality than those without glycemia [1]. DCM is featured by ventricular diastolic and (or) systolic dysfunction occurring in patients with type 1 or type 2 diabetes independent of hypertension, coronary artery disease (CAD), and other cardiovascular diseases [2]. The pathogenesis of DCM is a multistep process, which is implicated with the alterations of various vital events, including mitochondrial dysfunction, altered lipid metabolism, endoplasmic reticulum stress, oxidative stress, inflammation, and epigenetic changes $[3,4]$. Evidence is mounting that the occurrence and progression of DCM are triggered by the abnormal expression or mutation of genes, such as S6 kinase 1 (S6K1) [5], CD36 [6], peroxisome proliferator-activated receptor- $\alpha$ (PPAR- $\alpha$ ) [7], and protein kinase $\mathrm{C}(\mathrm{PKC})$ [8]. Currently, the diagnosis of DCM in clinics mainly relies on the serum natriuretic peptide (NAPP) level and other noninvasive tests involving electrocardiography, to clarify ventricular overload; X-ray, to evaluate fluid accumulation; and conventional cardiac ultrasound, to figure out cardiac structure and function. However, these methods for DCM diagnosis lack the specificity and efficiency; it was thus difficult for us to obtain early and 
accurate diagnosis as well as treatment, so some patients with DCM missed the best opportunity for treatment, thereby increasing death risk [1]. Hence, identifying the specific and sensitive genes or proteins which can help us confirm the patients with DCM as early as possible is of vital significance, not only for more accurate diagnosis, better treatment, and ideal prognosis but also for an overall understanding of the molecular mechanisms underlying DCM.

Bioinformatic analysis and gene expression profiling analysis have enabled us to screen molecular markers among healthy individuals and patients, which provides novel insights into diseases at multiple levels ranging from the alterations of copy number at the genome level to gene expression at transcriptome level, and even epigenetic alterations. However, in fact, the application of these microarrays has not gained popularity as expected in clinics because of an overwhelming amount of genes identified by gene profiling, lack of validation or repeatability, and intricate statistical analyses [9-11]. Therefore, for the purpose of putting these expression profiles in clinical practice as quickly as possible, it is of necessity to validate a suitable amount of genes and develop a proper and ideal approach which could be operated routinely.

In the current study, the gene expression profile of GSE26887 was downloaded from the Gene Expression Omnibus (GEO, http://www.ncbi.nlm.nih.gov/geo/) and analyzed using the GEO2R online tool to comprehensively identify the differentially expressed genes (DEGs) between DCM and healthy individuals. Furthermore, we also analyzed the gene ontology (GO) involving biological process (BP), molecular function (MF), cellular component (CC), and KEGG pathways of these DEGs. Subsequently, we carried out a protein-protein interaction (PPI) network of these DEGs and chose the top 15 hub genes with a high degree of connectivity. Meanwhile, we also re-identified the top 15 hub genes by PCR and Western blot.

\section{Materials and Methods}

2.1. Microarray Data. We obtained the microarray of GSE26887 from the National Center for Biotechnology Information (NCBI) GEO database, which is a free and publicly available database [10]. The GSE26887 dataset possesses 24 samples in all, containing 5 normal individuals, 7 patients with diabetic cardiomyopathy, and 12 nondiabetic-heart failure patients with ischemic cardiomyopathy, which was based on the GPL6244 platform [HuGene-1_0-st] Affymetrix Human Gene 1.0 ST Array [transcript (gene) version] by Greco et al. We also downloaded the Series Matrix File of GSE26887 from the GEO database in Pubmed. All of these cardiac tissues were acquired from the vital, noninfarcted zone derived from patients with dilated hypokinetic postischemic cardiomyopathy in surgical ventricular restoration. Inclusion criteria of the diabetic group for this microarray were blood glucose $\geq 126 \mathrm{mg} / \mathrm{dL}$, previous type 2 diabetes mellitus (T2DM) diagnosis, or receiving antidiabetic therapy, and those for the nondiabetic group were blood glucose $<100 \mathrm{mg} / \mathrm{dL}$ and HbAlc n.v. 4.8-6.0\%. Additionally, heart failure patients were matched for ejection fraction (LVEF), end systolic volume (ESV), sex, age, smoke habits, ethnic distribution, body mass index (BMI), hypertension, and glomerular filtration rate. The gene expression profile was assessed by Affymetrix GeneChip Human Gene 1.0 ST array using total RNA extracted from the above samples.

2.2. Identification of DEGs. We screened the DEGs between DCM and healthy samples using GEO2R (http://www.ncbi. nlm.nih.gov/geo/geo2r), an interactive analysis tool for the GEO database on the basis of $\mathrm{R}$ language. Consistent with the previous criteria $[12,13]$, we defined the genes with $\log$ $\mathrm{FC}<-1$ (downregulated genes) or $\log \mathrm{FC}>1$ (upregulated genes) as differentially expressed. Meanwhile, the adjusted $P$ value $<0.05$ was regarded statistically different, aiming at reducing the false positive rate. Furthermore, after downloading the relatively raw TXT data, we also applied visual hierarchical cluster analysis to display the volcano plot and heat map of DCM and healthy samples using ImageGP (http://www.ehbio.com/ImageGP/index.php/Home/Index/ index.html).

2.3. Protein-Protein Interaction (PPI) Network. The PPI network could identify the core hub genes and key gene modules between healthy individuals and patients [14]. Firstly, we used the Search Tool for the Retrieval of Interacting Genes/ Proteins (STRING), which is a well-known database containing the predicted and recognized protein interactions (https://string-db.org/), to identify the PPI association. Subsequently, we applied Cytoscape software platform on the basis of the PPI associations to construct the PPI network. Top 15 hub genes were selected according to the ranking order of connectivity degree.

2.4. Gene Ontology (GO) and Kyoto Encyclopedia of Genes and Genomes (KEGG) Pathway Analysis. GO analysis can annotate a collection of genes with functions involving molecular function (MF), cellular components (CC), and biological process (BP) [15]. The KEGG pathway is a group of databases which could hint biological pathways of certain genes implicated with diseases and drugs. KEGG in essence is a resource for us to receive an integrated understanding of biological functions and even some advanced genome information [16]. The GO and KEEG analysis in our study was performed by the Database for Annotation, Visualization and Integrated Discovery (DAVID, http://david.ncifcrf.gov) (version 6.7), an online biological function database integrating considerable biological data and analysis tools [17]. $P<0.05$ should be the cut-off criterion. We also used ImageGP to construct the enrichment plots, aiming to visualize the BP, MF, CC, and KEGG pathways of these DEGs.

2.5. Animals. All animal experimental procedures in this study were approved by the Animal Care and Use Committee of Renmin Hospital of Wuhan University and were performed in accordance with the Care and Use of Laboratory Animals published by the US National Institute of Health (Revised 2011). Both male type 2 diabetic (db/db) $(n=8)$ and WT mice $(n=8)$ (8-10 weeks) weighing $25.2 \pm 2 \mathrm{~g}$ were purchased from the Institute of Laboratory Animal Science, Chinese Academy of Medical Sciences (Beijing, China). 
2.6. Quantitative Real-Time PCR ( $q P C R$ ). The mice were sacrificed by injecting excessive sodium pentobarbital. Whereafter, the left ventricles of mice were collected for further RNA detection. Total RNA was isolated using the TRIzol (Invitrogen, Carlsbad, CA, USA) assay, the concentrations and purities of which were quantified using an ultraviolet spectrophotometer. The RNA was then reversely transcribed according to the previous description [18]. The expression levels of top 5 upregulated genes and top 5 downregulated genes were normalized to GAPDH. Relative mRNA expression levels were analyzed by the $2^{-\Delta \Delta}$ cycle threshold (CT) method. The primer sequences are displayed in Table S1.

2.7. Western Blot. Protein extraction, SDS-PAGE, and immunodetection of the cardiac tissues were all performed according to our previous research. Protein expression levels were normalized to the matched total proteins or GAPDH [19].

2.8. Cell Culture and Treatment. Neonatal rat cardiomyocytes and neonatal rat cardiac fibroblasts were isolated according to the previous study [20]. Cardiomyocyte hypertrophy was evaluated by anti- $\alpha$-actinin immunofluorescence staining while the phenotypic change of cardiac fibroblasts was evaluated by anti- $\alpha$-SMA immunofluorescence staining. For cell transfection, replication-defective adenoviral vectors were employed to upregulate the expression of SOCS3. After infection, cardiomyocytes and cardiac fibroblasts were incubated with a high-glucose concentration ( $33 \mathrm{mM}$ glucose) while the normal group was exposed to a normal glucose concentration ( $5.5 \mathrm{mM}$ glucose).

2.9. Statistical Analysis. The obtained data were presented as mean \pm SD (standard deviation) and assessed by the twotailed Student's $t$-test. A difference of $P<0.05$ was considered statistically significant.

\section{Results}

3.1. Identification of DEGs. The overall flow diagram of our study is presented in Figure 1. In this study, a total of 5 normal individuals and 7 patients with DCM were analyzed. We applied the GEO2R online analysis tool with default parameters to screen the DEGs, using adjusted $P$ value $<0.05$ and $\log \mathrm{FC} \leq-1$ or $\log \mathrm{FC} \geq 1$ as the cut-off criteria. After analyzing GSE26887, 236 DEGs were captured, including 134 upregulated genes and 102 downregulated genes. The expression proportion of these DEGs is displayed in the volcano plot (Figure 2(a)). The heat map represented the top 25 upregulated genes and top 25 downregulated genes between patients with DCM and healthy individuals (Figure 2(b)). Among these $236 \mathrm{DEGs}$, the top 5 upregulated genes involved NPPA, SFRP4, DSC1, NEB, and FRZB while the top 5 downregulated genes were SERPINE1, SERPINA3, ANKRD2, XRCC4, and S100A8. The gene tiles and biological functions of these 10 genes are displayed in Table 1.

To ensure the credibility of the microarray of GSE26887 and obtain further credible analysis, we re-identified the top 5 upregulated genes and top 5 downregulated genes via qPCR in vivo and in vitro. The results of echocardiography, hematoxylin and eosin (H\&E) staining, and picrosirius red (PSR)

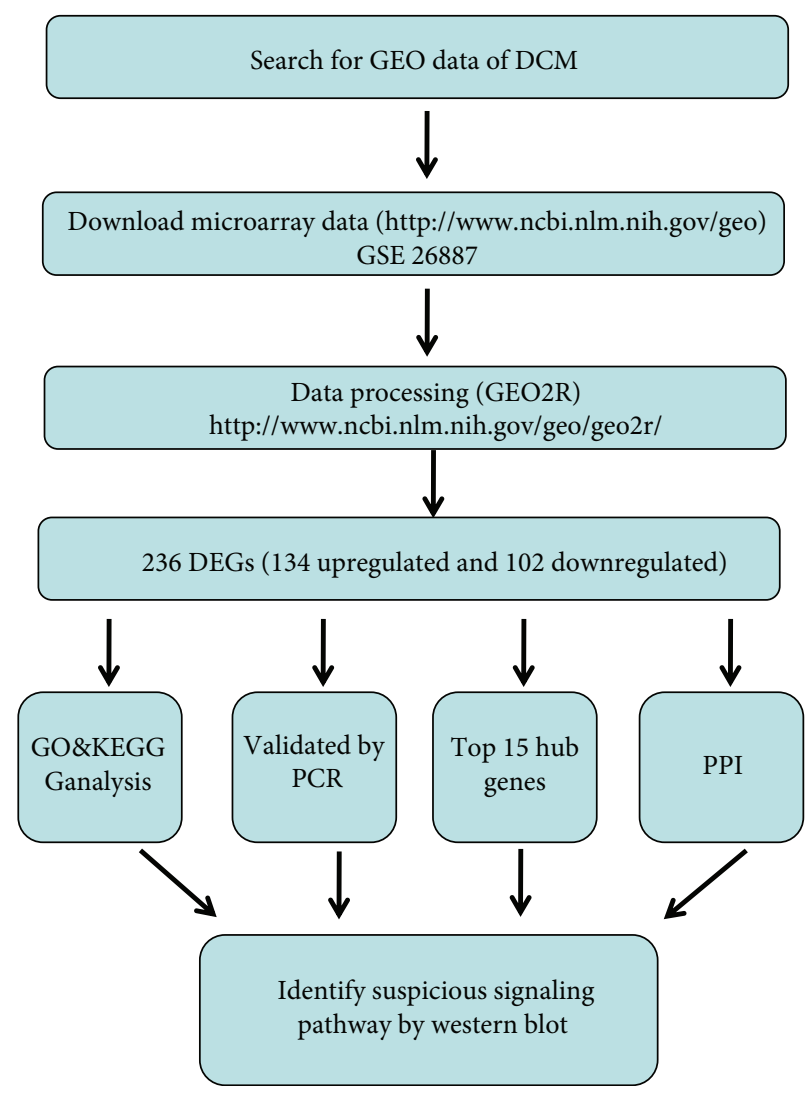

FIGURE 1: Flow diagram of the analysis procedure: data collection, preprocessing, analysis, and validation.

indicated that the DCM model of $\mathrm{db} / \mathrm{db}$ mice was constructed successfully (Figure S1A-B). The results from PCR demonstrated that the mRNA expression levels of NPPA, SFRP4, DSC1, NEB, and FRZB were significantly higher in the DCM group compared to the healthy group while the mRNA expression levels of SERPINE1, SERPINA3, ANKRD2, XRCC4, and S100A8 in the DCM group were statistically lower than those in the healthy group (Figures $3(\mathrm{a})-3(\mathrm{j})$ ). Also, we detected the expression levels of these genes in cardiomyocytes and cardiac fibroblasts, respectively. In cardiac fibroblasts, the alterations of the ten genes were consistent with the mouse model (Figure S2A-B). Intriguingly, the expression level of ANKRD2 in cardiomyocytes displayed no significant difference between the normal group and the HG group. In spite of this, other nine genes in cardiomyocytes had a similar variation trend with the mouse model and cardiac fibroblasts (Figure S2C-D). One the one hand, these results increased the credibility of this microarray. On the other hand, these DEGs with the most significant difference may be the promising candidates in clinics to diagnose DCM.

3.2. GO Enrichment Analysis. The results from GO term enrichment analysis varied from expression alterations and GO classification of these DEGs. By analyzing GO enrichment of these upregulated DEGs and downregulated DEGs via DAVID, we discovered that the upregulated DEGs in BP were mainly enriched in the G-protein-coupled purinergic 


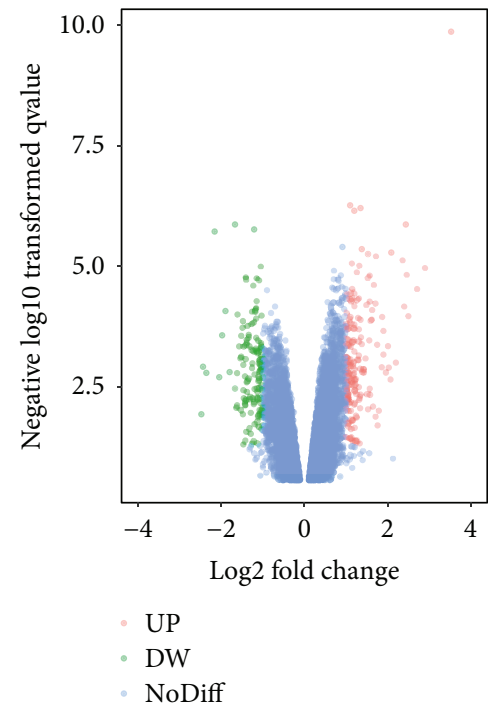

(a)

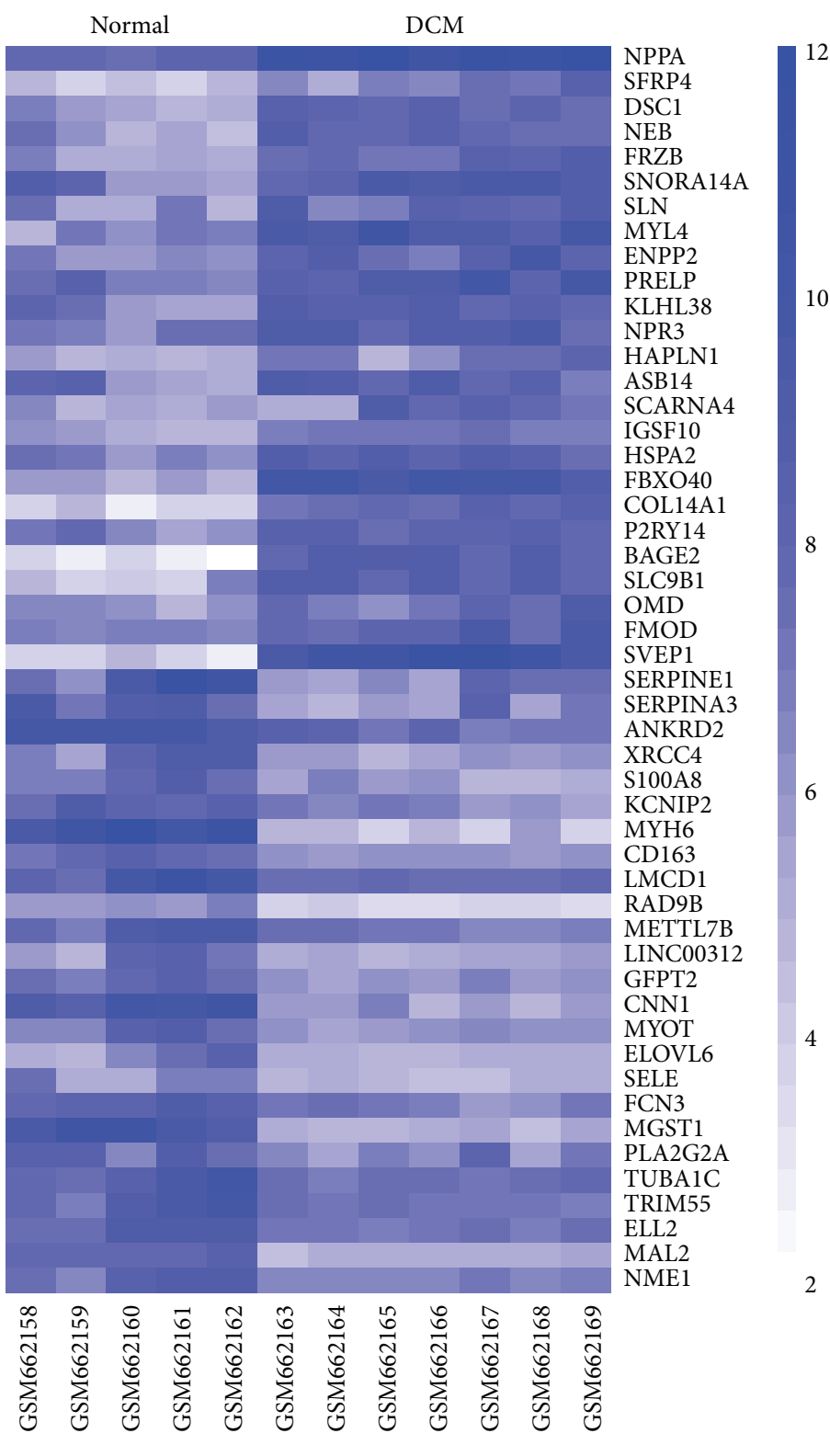

(b)

FIGURE 2: Volcano plot and heat map of the differentially expressed genes (DEGs) between normal samples and patients with diabetic cardiomyopathy (DCM). (a) Volcano plot of genes detected in DCM. Green means downregulated DEGs; red means upregulated DEGs; blue means no difference. (b) Heat map of top 25 upregulated DEGs and top 25 downregulated DEGs.

nucleotide receptor signaling pathway, fatty acid metabolism, mitochondrial membrane potential, extracellular matrix organization, and mitochondrial permeability transition while the downregulated DEGs in BP were enriched in inflammatory response, lipid intake, response to drug, immune response, and platelet degranulation. As for CC, the upregulated DEGs were principally enriched in the integral component of the membrane, plasma membrane, extracellular exosome, extracellular space, and extracellular region while the downregulated DEGs were enriched in the plasma membrane, extracellular space, extracellular region, extracellular exosome, and endoplasmic reticulum membrane. Additionally, MF analysis uncovered that the upregulated DEGs were principally enriched in zinc ion binding, calcium ion binding, heparin binding, collagen binding, and NADP binding. The downregulated genes were responsible for protein binding, mitochondrial uncoupling, cytokine activity, actin binding, and phosphatase activity (Table 2 and Figures 4(a)-4(c)).

3.3. KEGG Pathway Analysis. To acquire more comprehensive information regarding the vital pathways of those selected DEGs, KEGG pathways were also analyzed via DAVID. The results in Table 3 and Figure 4(d) unveiled 
TABLE 1: The top 5 upregulated and downregulated differentially expressed genes in patients with diabetic cardiomyopathy.

\begin{tabular}{lcccc}
\hline DEGs & Gene title & Gene symbol & LogFC & Biological function \\
\hline \multirow{4}{*}{ Upregulated } & Natriuretic peptide A & NPPA & 3.53 & Extracellular fluid volume and electrolyte homeostasis \\
& Secreted frizzled related protein 4 & SFRP4 & 2.71 & Soluble modulators of Wnt signaling \\
& Desmocollin 1 & DSC1 & 2.44 & Calcium-dependent glycoprotein \\
& Nebulin & NEB & 2.4 & Cytoskeleton \\
& Frizzled-related protein & FRZB & 2.36 & Soluble modulators of Wnt signaling \\
\hline \multirow{3}{*}{ Downregulated } & Serpin family E member 1 & SERPINE1 & -2.48 & Inhibitor of fibrinolysis \\
& Serpin family A member 3 & SERPINA3 & -2.44 & Anti-inflammatory and antioxidant effects \\
& Ankyrin repeat domain 2 & ANKRD2 & -2.16 & Modulator of NF- $\kappa$ B-mediated inflammatory \\
& X-ray repair cross-complementing 4 & XRCC4 & -2.05 & DNA repair \\
& S100 calcium-binding protein A8 & S100A8 & -1.98 & Regulating inflammation and oxidative stress, \\
& & & & activatingTLR4 signaling
\end{tabular}

the most important KEGG pathway of the downregulated and upregulated DEGs. The downregulated DEGs were mainly enriched in the PI3K-Akt signaling pathway, MAPK signaling pathway, HIF-1 signaling pathway, TNF signaling pathway, and Toll-like receptor signaling pathway. By contrast, the upregulated DEGs, namely, FMO4, FMO2, FMO3, ADH1B, and UGT2B4, had a strong correlation with drug metabolism-cytochrome P450.

3.4. PPI Analysis. Applying the STRING online tool, 120 nodes with 162 PPI relationships were identified, which accounted for approximately $90.3 \%$ of these selected DEGs (Figure 5(a)). Based on the degree of connectivity, we constructed the PPI network and selected the top 15 hub genes (Table 4). The top 15 hub genes, possessing high degree of connectivity in DCM, are as follows: IL6, MYC, ACTA2, SERPINE1, ASPN, SPP1, KIT, TFRC, FMOD, PDE5A, MYH6, FPR1, C3, CDKN1A, and SOCS3. Among these 15 hub genes, IL6, MYC, SERPINE1, SPP1, TFRC, MYH6, FPR1, C3, CDKN1A, and SOCS3 were significantly downregulated while ACTA2, ASPN, KIT, FMOD, and PDE5A were upregulated. The 15 hub genes could interact with 189 genes directly, and IL6 acted as the most intensive gene which could interact with 32 downregulated genes and 15 upregulated genes. Intriguingly, among these, hub genes also displayed strong interactions (Figure 5(b)). For instance, ACTA2 could directly interact with multiple genes (FMOD, IL6, MYH6, MYC, and ASPN), and SPP1 interacted with 4 hub genes (KIT, IL6, MYC, and SERPINE1). The details of the interactions among these 15 hub genes are shown in Table 5. Taken together, these results suggested that these hub genes, especially IL6, ACTA2 as well as SPP1 may exert critical effects in DCM.

3.5. Functional Analysis. To figure out the role of the IL6/STAT3/SOCS3 signaling pathway in the development of DCM, we detected the protein expression levels of SCOS3, phosphorylated-STAT3 (P-STAT3), and total STAT3 between the normal group and the $\mathrm{db} / \mathrm{db}$ group. The results showed that P-STAT3 had a significantly higher expression level in the DCM group compared to the normal group. Meanwhile, the level of SCOS3 was significantly downregulated in the DCM group (Figure 6). To further explore the role of the IL-6/STAT3/SOCS3 signaling pathway in cardiomyocytes and cardiac fibroblasts stimulated by HG, we firstly detected the mRNA expression of SOCS3 in cardiomyocytes and cardiac fibroblasts. As expected, the levels of SOCS3 in both cardiomyocytes and cardiac fibroblasts were significantly lower in the HG group compared with the normal group (Figures $7(\mathrm{a})$ and $7(\mathrm{~b})$ ). Additionally, immunofluorescent staining showed that the hypertrophic reactions of cardiomyocytes and phenotypic switching of cardiac fibroblasts were significantly abolished after SOCS3 was upregulated (Figures $7(\mathrm{c})$ and $7(\mathrm{~d})$ ). Meantime, hypertrophic markers and fibrotic markers were also decreased by the overexpression of SOCS3, evidenced by the lower levels of ANP, BNP, collagen I, and collagen III (Figures 7(e) and $7(\mathrm{f})$ ). Western blot showed that the overexpression of SOCS3 could inhibit the phosphorylation of STAT3 (Figures $7(\mathrm{~g})$ and $7(\mathrm{~h}))$.

\section{Discussion}

Diabetes mellitus has been broadly regarded as one of the leading causes of morbidity and mortality for several decades worldwide. According to estimates, by 2030, there will be approximately 450 million persons with diabetes. DCM serves as the major etiological factor and death cause of patients with diabetes, the incidence of which has increased over recent years [21]. However, currently, there is no specific and efficient diagnostic methodology and treatment strategy for DCM, which is partially because of the complicated molecular mechanisms, as well as its being asymptomatic for the first several years [2]. Hence, some key diagnostic biomarkers and therapeutic targets in plasma and myocardial biopsy should be verified as early as possible. Although myocardial biopsy is not as routine as that in tumors, it does not mean that it makes no sense to perform myocardial biopsy. Takeishi and Yoshihisa retrospectively analyzed 378 patients with suspected cardiomyopathy who underwent myocardial biopsy and found that the diagnostic impact of myocardial biopsy may be relatively high in patients with suspected hypertrophic cardiomyopathy compared to those with suspected dilated cardiomyopathy [22]. Additionally, in patients with 


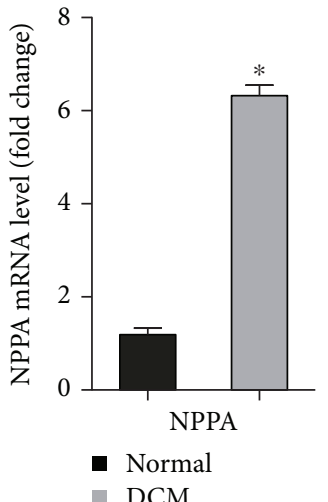

(a)

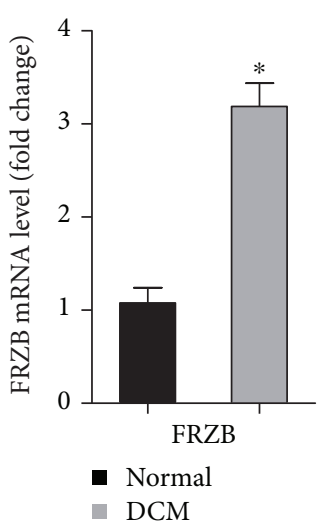

(e)

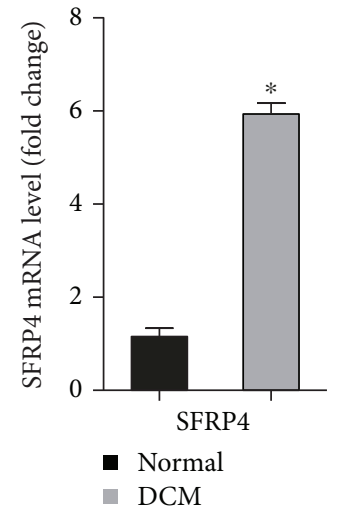

(b)

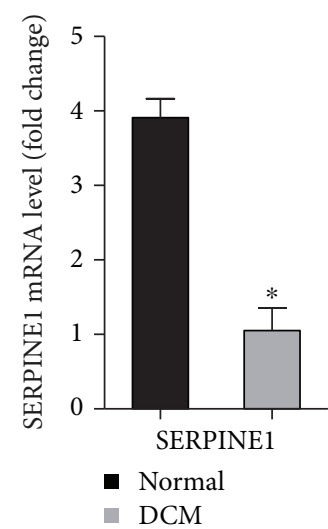

(f)

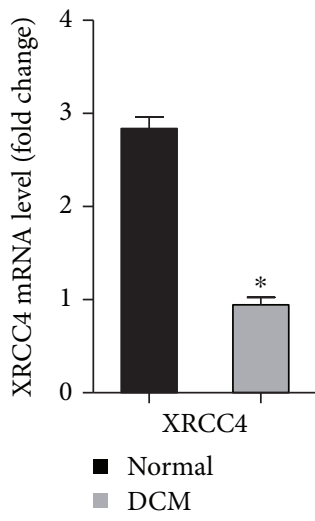

(i)

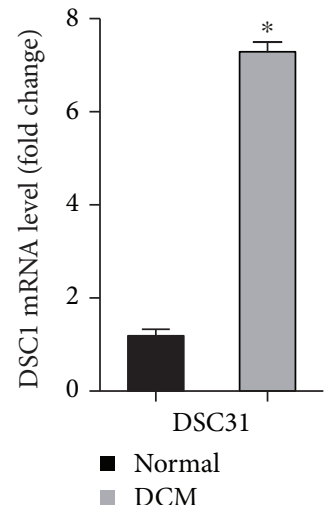

(c)

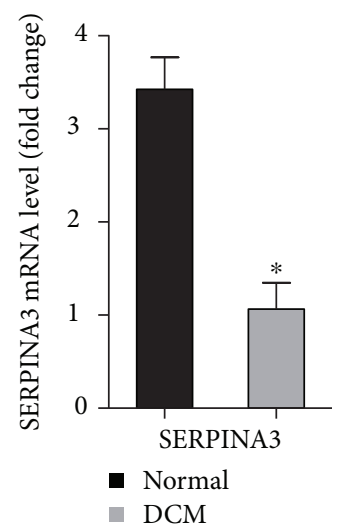

(g)

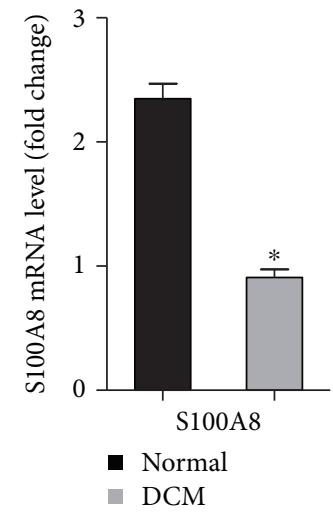

(j)

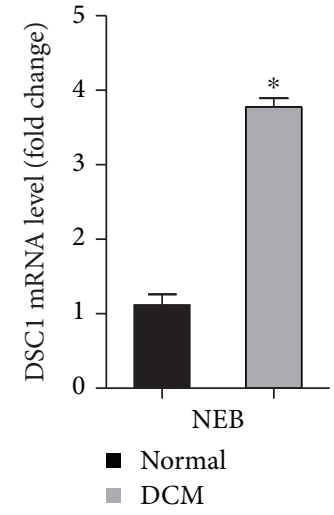

(d)

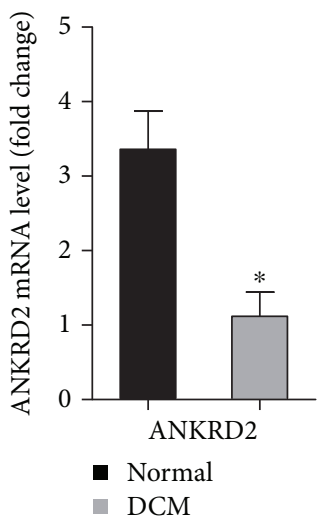

(h)

FIGURE 3: Validation of top 5 upregulated and top 5 downregulated DEGs in the mouse model of DCM. (a-e) NPPA, SFRP4, DSC31, NEB, and FRZB were significantly upregulated in the DCM group. (f-j) SERPINE1, SERPINA3, ANKRD2, ANKRD2, and S100A8 were significantly downregulated in the DCM group. ${ }^{*} P<0.05$ versus normal group.

arrhythmogenic right ventricular cardiomyopathy (ARVC), Yoshida et al. detected the expression of plakoglobin and connexin 43 in myocardial biopsy specimens and confirmed the correlations between the levels of these 2 proteins and the development of ARVC, indicating that plakoglobin and connexin 43 are two specific biomarkers of arrhythmic events in ARVC [23]. Furthermore, the combination of cardiac magnetic resonance (CMR) imaging and myocardial biopsy may also improve the diagnostic value in the evaluation of cardiomyopathic conditions [24]. The above studies further supported the potential of myocardial biopsy in diagnosis of DCM. In this study, we firstly performed a comprehensive investigation on expression profiling of myocardial tissue obtained from patients with DCM. Our study included 5 normal individuals and 7 patients with DCM from the GEO database of GSE26887. In our analysis, a total of 236 DEGs (accounting for $2.6 \%$ of all genes) were found, involving 134 upregulated genes and 102 downregulated genes. By 
TABLE 2: Gene ontology analysis of differentially expressed genes in patients with diabetic cardiomyopathy.

\begin{tabular}{|c|c|c|c|c|}
\hline Expression & Category & Term & Count & $\%$ \\
\hline \multirow{15}{*}{ Upregulated } & GOTERM_BP_DIRECT & $\begin{array}{l}\text { GO:0035589 G-protein-coupled purinergic } \\
\text { nucleotide receptor signaling pathway }\end{array}$ & 9 & 0.05 \\
\hline & GOTERM_BP_DIRECT & GO:0007155 fatty acid metabolism & 8 & 0.05 \\
\hline & GOTERM_BP_DIRECT & GO:0001501 mitochondrial membrane potential & 6 & 0.04 \\
\hline & GOTERM_BP_DIRECT & GO:0030198 extracellular matrix organization & 6 & 0.04 \\
\hline & GOTERM_BP_DIRECT & GO:0007409 mitochondrial permeability transition & 5 & 0.03 \\
\hline & GOTERM_CC_DIRECT & GO:0016021 integral component of membrane & 42 & 0.25 \\
\hline & GOTERM_CC_DIRECT & GO:0005886 plasma membrane & 37 & 0.22 \\
\hline & GOTERM_CC_DIRECT & GO:0070062 extracellular exosome & 29 & 0.17 \\
\hline & GOTERM_CC_DIRECT & GO:0005615 extracellular space & 22 & 0.13 \\
\hline & GOTERM_CC_DIRECT & GO:0005576 extracellular region & 20 & 0.11 \\
\hline & GOTERM_MF_DIRECT & GO:0008270 glucose transporter & 13 & 0.08 \\
\hline & GOTERM_MF_DIRECT & GO:0005509 calcium ion binding & 11 & 0.07 \\
\hline & GOTERM_MF_DIRECT & GO:0008201 heparin binding & 7 & 0.05 \\
\hline & GOTERM_MF_DIRECT & GO:0005518 collagen binding & 5 & 0.03 \\
\hline & GOTERM_MF_DIRECT & GO:0050661 NADP binding & 4 & 0.03 \\
\hline \multirow{15}{*}{ Downregulated } & GOTERM_BP_DIRECT & GO:0006954 inflammatory response & 11 & 0.07 \\
\hline & GOTERM_BP_DIRECT & GO:0008284 lipid intake & 9 & 0.06 \\
\hline & GOTERM_BP_DIRECT & GO:0042493 response to drug & 6 & 0.04 \\
\hline & GOTERM_BP_DIRECT & GO:0006955 immune response & 6 & 0.04 \\
\hline & GOTERM_BP_DIRECT & GO:0002576 platelet degranulation & 5 & 0.04 \\
\hline & GOTERM_CC_DIRECT & GO:0005886 plasma membrane & 31 & 0.21 \\
\hline & GOTERM_CC_DIRECT & GO:0005615 extracellular space & 23 & 0.15 \\
\hline & GOTERM_CC_DIRECT & GO:0005576 extracellular region & 21 & 0.14 \\
\hline & GOTERM_CC_DIRECT & GO:0070062 extracellular exosome & 21 & 0.14 \\
\hline & GOTERM_CC_DIRECT & GO:0005789 endoplasmic reticulum membrane & 13 & 0.09 \\
\hline & GOTERM_MF_DIRECT & GO:0005515 protein binding & 58 & 0.39 \\
\hline & GOTERM_MF_DIRECT & GO:0008083 mitochondrial uncoupling & 5 & 0.03 \\
\hline & GOTERM_MF_DIRECT & GO:0005125 cytokine activity & 5 & 0.03 \\
\hline & GOTERM_MF_DIRECT & GO:0003779 actin binding & 5 & 0.03 \\
\hline & GOTERM_MF_DIRECT & GO:0016791 phosphatase activity & 3 & 0.03 \\
\hline
\end{tabular}

GO: gene ontology.

further annotating and analyzing these DEGs, we identified 10 sensitive biomarkers and top 15 hub genes among these DEGs. Additionally, we also speculated the putative mechanisms of SOCS3 contributing to DCM by Western blot.

4.1. The Production of IL6 Is Essential for the Development of DCM. IL6 is a critical cytokine exerting multiple physiological effects in inflammation and immune regulation, which could be secreted by a range of cell types including monocytes, mast cells, lymphocytes, macrophages, endothelial cells, keratinocytes, tumor cell lines, and fibroblasts [25]. In the innate immune system and adaptive immunity, IL6 stimulation could trigger different biological activities [26]. For example, in innate immunity, IL6 could accelerate the production of neutrophils as well as the synthesis of acutephase proteins, thus giving rise to acute-phase response while in adaptive immunity, IL6 stimulation could increase the proliferation of $B$ cells [27]. Notably, IL-6 pretreatment increased collagen production in cultured cardiac fibroblasts and promote interstitial fibrosis in Ang II-induced rat heart $[28,29]$. Zhang et al. demonstrated that deletion of IL-6 preserved cardiac function and mitigated the interstitial fibrosis in streptozotocin-induced diabetic mice, the mechanism of which may involve the inhibitory effects of IL- 6 on TGF $\beta 1$ and miR-29 pathway [30]. Clinical trials also disclosed a strong correlation between elevated levels of circulating IL6 and heart failure severity and mortality in patients [31]. In our study, IL6 was an upregulated hub gene with the highest connectivity degree, indicating that IL6 may exert a core and predominant role in the development of DCM. Additionally, according to KEGG analysis, IL6 was significantly enriched in the PI3K/Akt signaling pathway, hypoxia-inducible factor-1 (HIF-1) signaling pathway, TNF signaling pathway, and Toll-like receptor signaling pathway. The previous study has demonstrated that HIF-1 deregulation during the early stage of diabetes gave rise to the development of DCM 


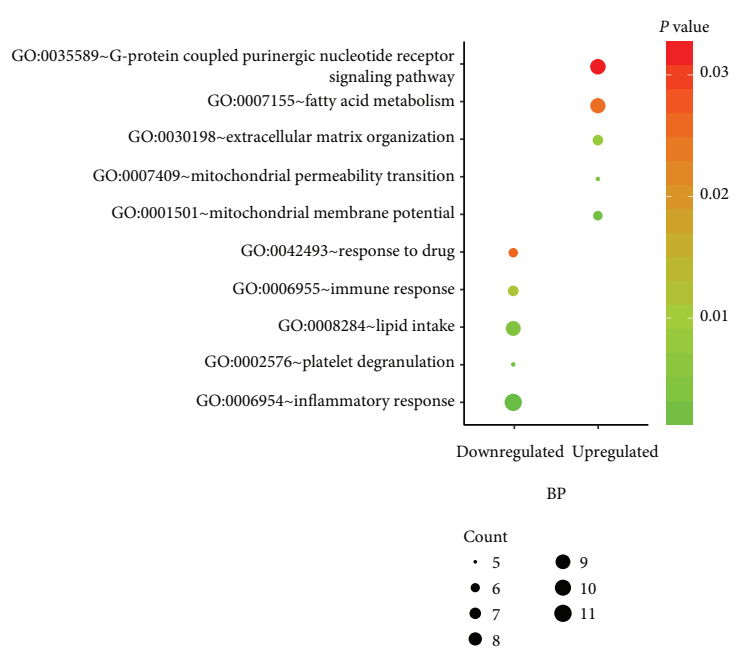

(a)

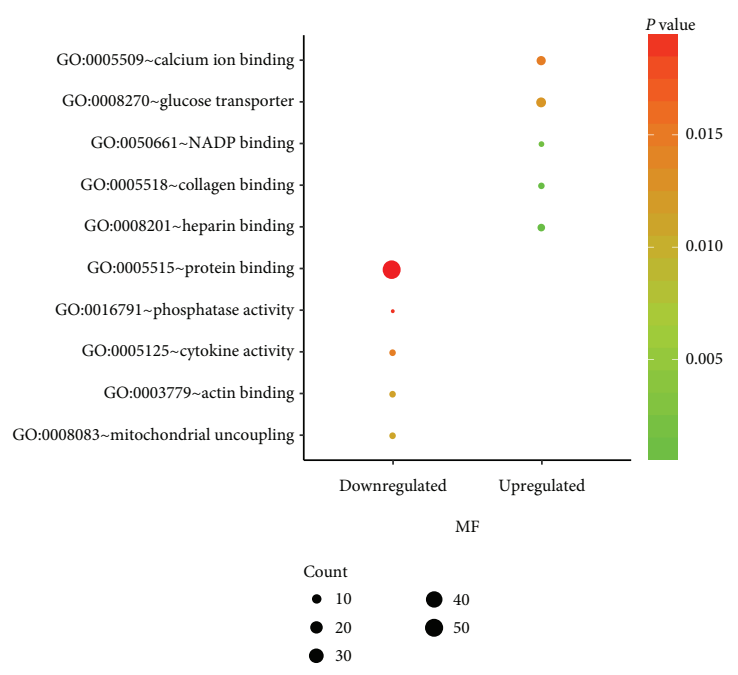

(c)

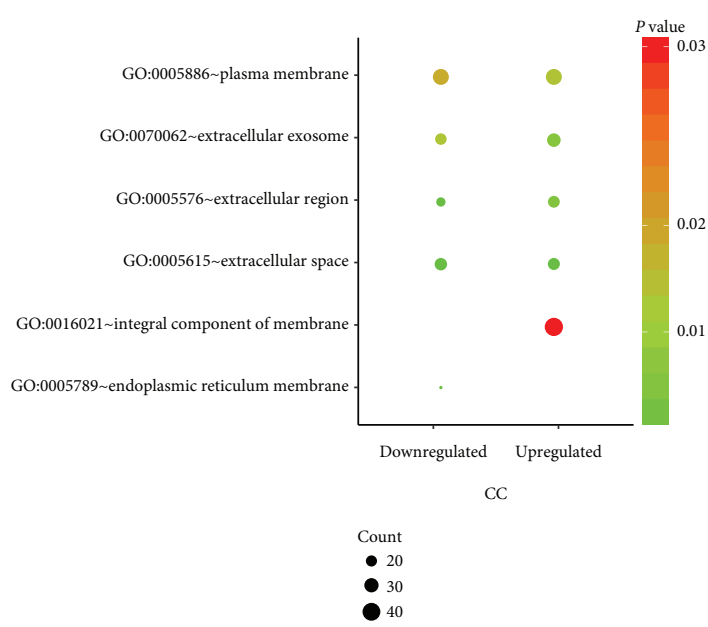

(b)

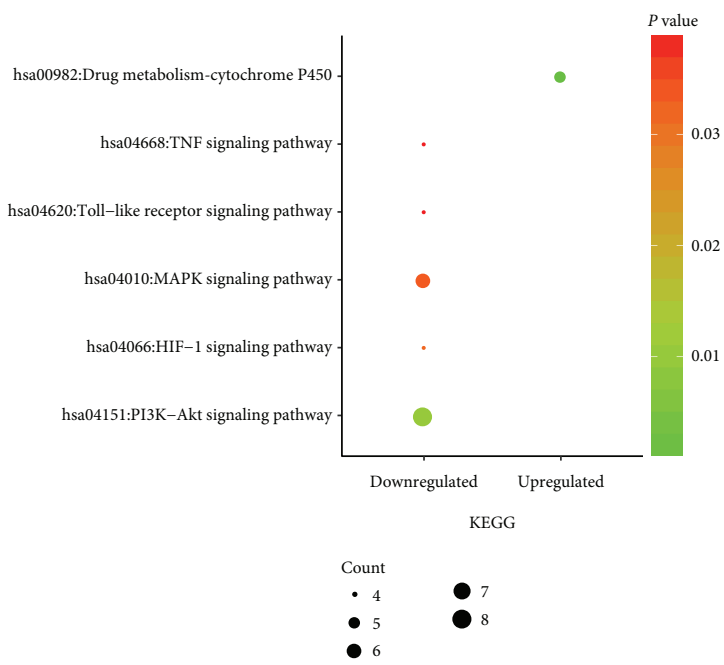

(d)

Figure 4: Gene ontology (GO) and Kyoto Encyclopedia of Genes and Genomes (KEGG) pathway analysis of DCM. (a) The enriched GO terms in the biological process (BP); (b) the enriched GO terms in the cellular component (CC); (c) the enriched GO terms in the molecular function (MF); (d) the enriched KEGG pathway in DCM.

TABLE 3: KEGG pathway analysis of differentially expressed genes in patients with diabetic cardiomyopathy.

\begin{tabular}{|c|c|c|c|c|c|}
\hline Category & Term & Count & $\%$ & $P$ value & Genes \\
\hline \multirow{5}{*}{ Downregulated DEGs } & hsa04151:PI3K-Akt signaling pathway & 8 & 0.05 & 0.01 & $\begin{array}{l}\text { FGF18, CDKN1A, IL6, FGF7, } \\
\text { TNC, LAMC2, MYC, and SPP1 }\end{array}$ \\
\hline & hsa04010:MAPK signaling pathway & 6 & 0.04 & 0.03 & $\begin{array}{c}\text { DUSP5, FGF18, FGF7, MAP2K3, } \\
\text { FLNC, and MYC }\end{array}$ \\
\hline & hsa04066:HIF-1 signaling pathway & 4 & 0.02 & 0.03 & CDKN1A, IL6, and TFRC \\
\hline & hsa04668:TNF signaling pathway & 4 & 0.02 & 0.04 & IL6, SOCS3, MAP2K3, and SELE \\
\hline & hsa04620:Toll-like receptor signaling pathway & 4 & 0.02 & 0.04 & IL6, LY96, MAP2K3, and SPP1 \\
\hline Upregulated DEGs & hsa00982:Drug metabolism-cytochrome P450 & 5 & 0.03 & 0.001 & $\begin{array}{l}\text { FMO4, FMO2, FMO3, } \\
\text { ADH1B, and UGT2B4 }\end{array}$ \\
\hline
\end{tabular}

KEGG: Kyoto Encyclopedia of Genes and Genomes; FDR: false discovery rate.

[32]. In diabetic retinopathy, the expression of proinflammatory IL6 and TNF- $\alpha$ were significantly inhibited after decreasing the expression of HIF-1 [33]. Hence, whether
IL6 could be effectively suppressed via blocking HIF-1 in DCM, eventually alleviating inflammation in myocardium, needs to be further explored. 


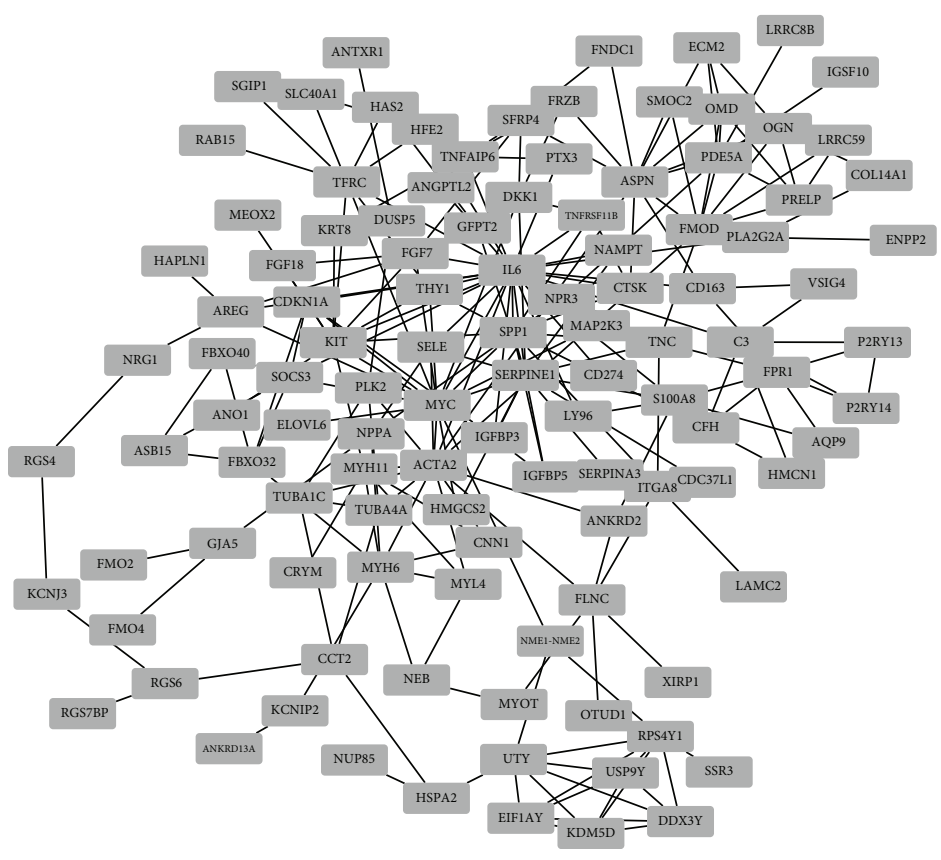

(a)

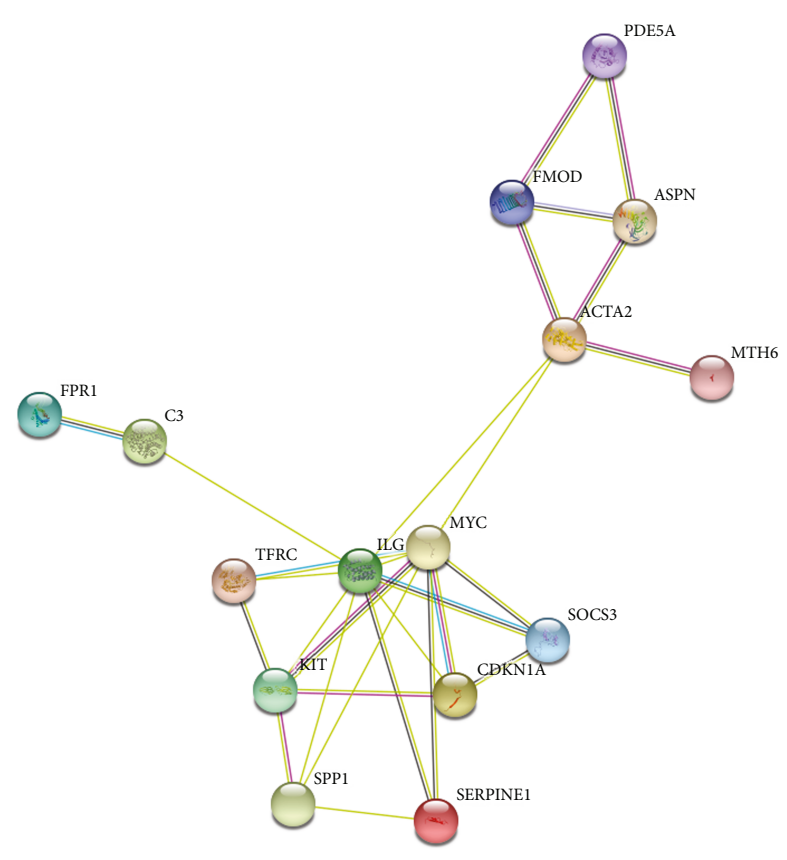

(b)

FIGURE 5: Protein-protein interaction (PPI) network. (a) The PPI network of overall DEGs and (b) the PPI network of top 15 hub genes with high connectivity degree.

TABle 4: Top 15 hub genes with higher degree of connectivity.

\begin{tabular}{lcc}
\hline Gene & Degree of connectivity & $P$ value \\
\hline IL6 & 29 & $5.48 E-04$ \\
MYC & 17 & $3.99 E-03$ \\
ACTA2 & 14 & $9.78 E-06$ \\
SERPINE1 & 14 & $1.16 E-02$ \\
ASPN & 12 & $9.15 E-03$ \\
SPP1 & 11 & $4.04 E-02$ \\
KIT & 11 & $4.54 E-03$ \\
TFRC & 9 & $3.34 E-04$ \\
FMOD & 9 & $3.42 E-04$ \\
PDE5A & 9 & $2.96 E-04$ \\
MYH6 & 8 & $1.55 E-03$ \\
FPR1 & 8 & $1.71 E-06$ \\
C3 & 7 & $1.18 E-02$ \\
CDKN1A & 7 & $3.94 E-04$ \\
SOCS3 & 7 & $1.02 E-03$ \\
\hline
\end{tabular}

4.2. Inflammation and Immune Disorders Are Vital Pathophysiological Alterations of DCM. Another major finding of our study is that the inflammation and immune disorders mediated by cytokines may exert an important role in DCM [34]. Cardiac inflammation is one of the important features in heart failure. Enhanced proinflammatory cytokine expression levels and intensified immune cell infiltration, including macrophages and cytotoxic $\mathrm{T}$ lymphocytes, have been previously observed in the inflamed heart in DCM
[35]. The activation of certain molecular pathways including c-Jun NH2-terminal kinase, NF- $\kappa \mathrm{B}$, and p38-MAPK could aggravate the development of inflammation which displayed a strong relationship with insulin resistance, thus inducing DCM [36-38]. In the diabetic hearts of type 2 diabetes patients, elevated inflammatory cytokines, such as IL6, TNF- $\alpha$, cell adhesion molecules, including vascular cell adhesion molecule-1 (VCAM-1) and intracellular adhesion molecule-1 (ICAM-1), and acute phase reactants, such as C-reactive protein and other inflammatory markers, have been verified $[39,40]$. On the other hand, immune disorder in cardiovascular diseases has been also studied for decades. Particularly, activation of the immune system is not independent of inflammation in the progression of heart failure. In chronic heart failure, activating the immune system can usually contribute to the activation of the complement system, secretion of proinflammatory cytokines, and production and release of autoantibodies [41]. As for DCM, impaired systolic and diastolic LV function in the streptozotocin-induced diabetes, to a great extent, was correlated with increased immune cell invasion and adverse cardiac remodeling [42]. Toll-like receptors (TLRs) are a type of membrane-anchored proteins existing in various cell types involving immune cells (macrophages and lymphocytes) and nonimmune cells (cardiomyocytes) $[43,44]$. Cardiac TLRs and inflammasome could interact with each other, inducing inflammation through reactive oxygen species overproduction and $\mathrm{NF}-\kappa \mathrm{B}$ activation [45]. In our study, the enriched Toll-like receptor signaling pathway was observed from KEGG analysis, indicating the vital role of immunity in DCM. Meanwhile, GO analysis unveiled that the downregulated DEGs were mainly enriched 
TABLe 5: Gene-specific primers used in quantitative real-time PCR.

\begin{tabular}{|c|c|c|c|}
\hline Species & Genes & & Sequences \\
\hline \multirow[b]{2}{*}{ Mouse } & \multirow{2}{*}{ GAPDH } & Forward & $5^{\prime}$-ACTCCACTCACGGCAAATTC-3' \\
\hline & & Reverse & $5^{\prime}$-TCTCCATGGTGGTGAAGACA-3' \\
\hline \multirow{2}{*}{ Mouse } & \multirow{2}{*}{ NPPA } & Forward & $5^{\prime}$-CCCTCCGATAGATCTGCCCT-3' \\
\hline & & Reverse & $5^{\prime}$-GTCAATCCTACCCCCGAAGC-3' \\
\hline \multirow{2}{*}{ Mouse } & \multirow{2}{*}{ SFRP4 } & Forward & $5^{\prime}$-AAAAGCCGTCCAGAGGAGTG-3' \\
\hline & & Reverse & 5'-GAGGGACTTGTGTTCGAGGG-3' \\
\hline \multirow{2}{*}{ Mouse } & \multirow{2}{*}{ DSC31 } & Forward & $5^{\prime}$-GATCAGGCCAGTGGAAATGT-3' \\
\hline & & Reverse & 5'-GTGTGTTTCGTGCAACCATC-3' \\
\hline \multirow{2}{*}{ Mouse } & \multirow{2}{*}{ NEB } & Forward & $5^{\prime}$-ATCCTGTCCAAACTAAGGCTCG-3' \\
\hline & & Reverse & $5^{\prime}$-ACCTCTTTAGCATAGTAGTCCGC-3' \\
\hline \multirow{2}{*}{ Mouse } & \multirow{2}{*}{ SERPINE1 } & Forward & 5'-GGGTTCACTTTACCCCTCCG-3' \\
\hline & & Reverse & $5^{\prime}$-TAGGGCAGTTCCACAACGTC-3' \\
\hline \multirow{2}{*}{ Mouse } & \multirow{2}{*}{ SERPINA3 } & Forward & 5'-TGACCTTTCTCAGCACGACC-3' \\
\hline & & Reverse & $5^{\prime}$-AATAGGGGAGGATGGGAGCA-3’ \\
\hline \multirow{2}{*}{ Mouse } & \multirow{2}{*}{ ANKRD2 } & Forward & $5^{\prime}$-TTGCCCAGGAGGAAGAGACT-3' \\
\hline & & Reverse & 5'-TGTCTCTCACGTTGGTGTCG-3’ \\
\hline \multirow{2}{*}{ Mouse } & \multirow{2}{*}{$\mathrm{XRCC} 4$} & Forward & $5^{\prime}$-TTGGGCGCATCGGTTTATCT-3' \\
\hline & & Reverse & 5'-ACCAGTGCCTTTCTCAGCTC-3' \\
\hline \multirow{2}{*}{ Mouse } & \multirow{2}{*}{ S100A8 } & Forward & $5^{\prime}$-TTCGTGACAATGCCGTCTGA-3' \\
\hline & & Reverse & $5^{\prime}$-GGCCAGAAGCTCTGCTACTC-3' \\
\hline
\end{tabular}

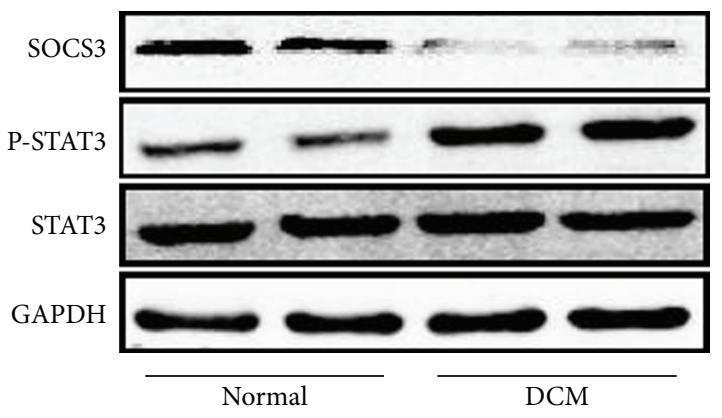

(a)

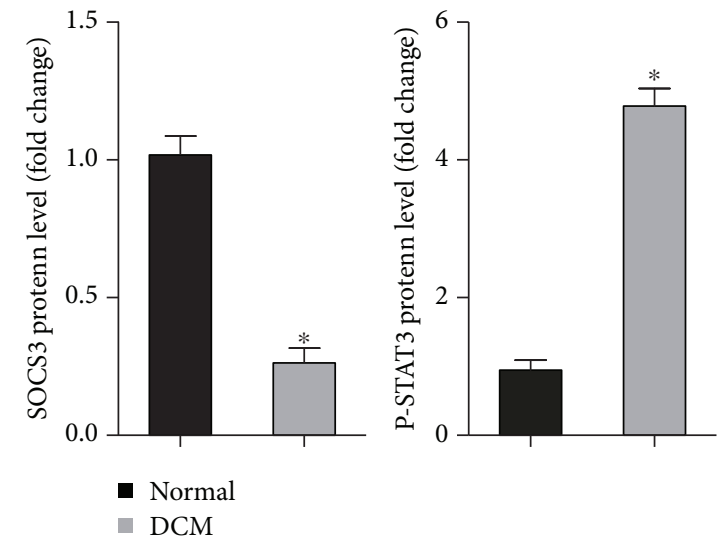

(b)

FIgURE 6: Identification of the STAT3/SOCS3 pathway in an in vivo model of DCM. SOCS3: phosphorylated-STAT3 (P-STAT3) and total STAT3 protein levels as shown by Western blot analysis.

in inflammatory response and immune response. Additionally, we found that the upregulated DEGs were significantly enriched in the G-protein-coupled purinergic nucleotide receptor signaling pathway in BP. To our knowledge, the nucleotides, the fundamental subunits of nucleic acids, are released by mast cells, macrophages, T cells, endothelial cells, and platelets in response to physiological activation. The purinergic nucleotide receptor could inhibit the activation of effector $\mathrm{T}$ cells in many allergic diseases [46]. Thus, we hypothesize that balancing the immune homeostasis by regulating the nucleotide receptor signaling pathway may be promised to be a novel strategy to treat DCM. Last but not 


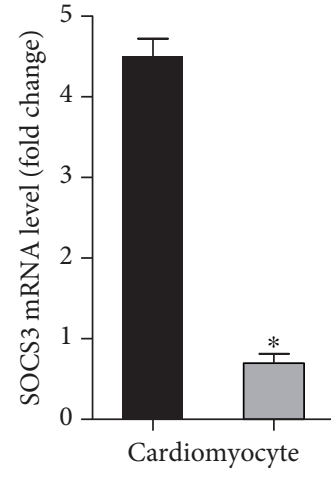

- Normal

- $\mathrm{HG}$

(a)

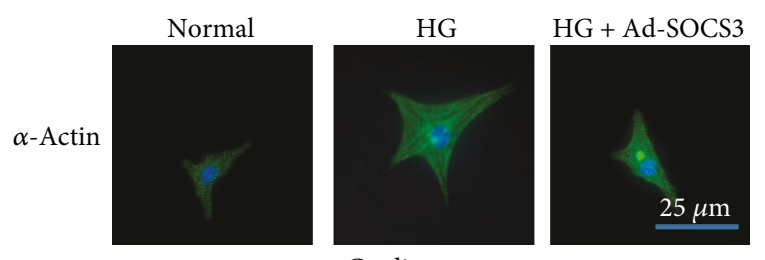

Cardiomyocyte

(c)
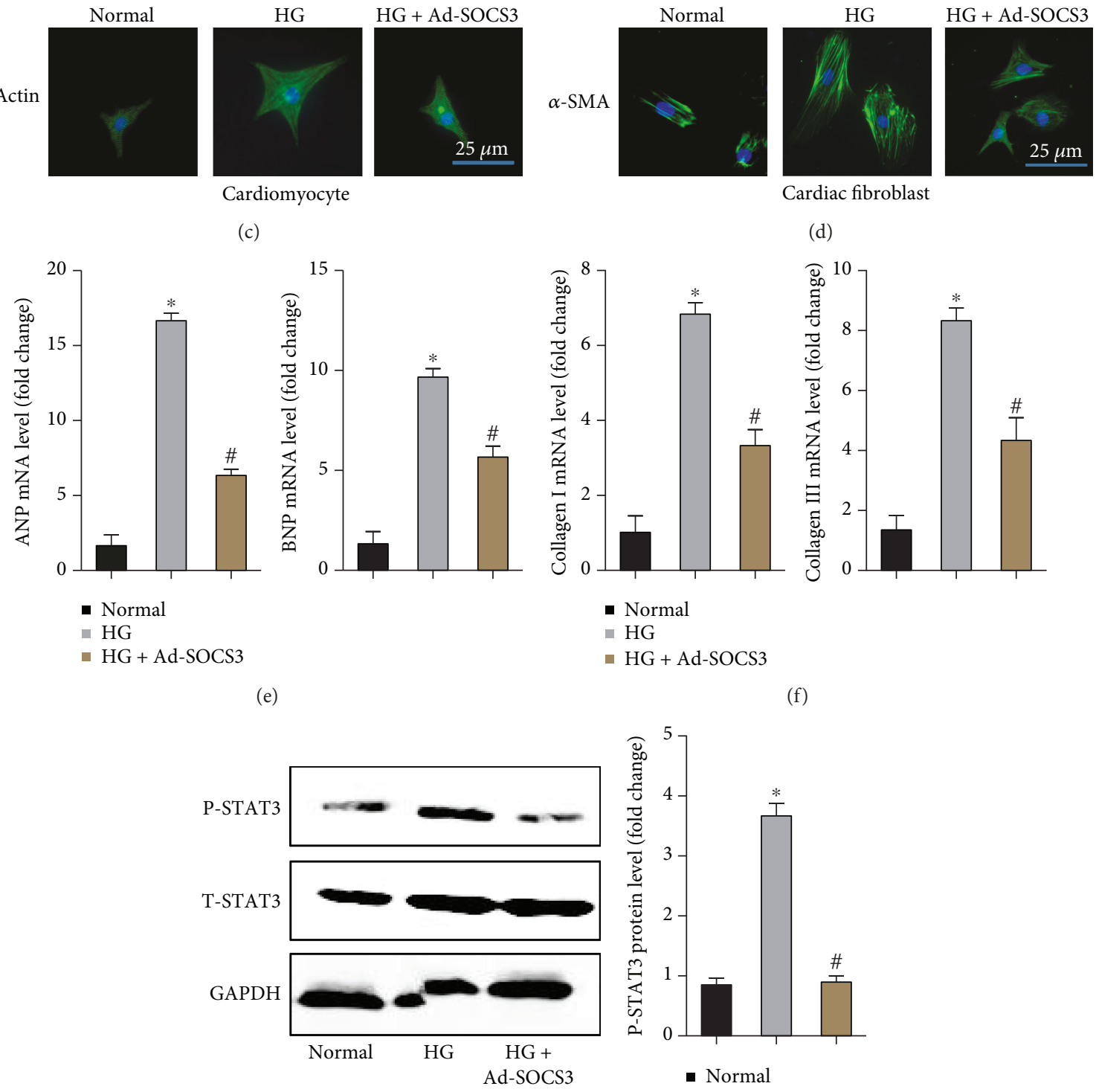

Cardiac fibroblast

(d)

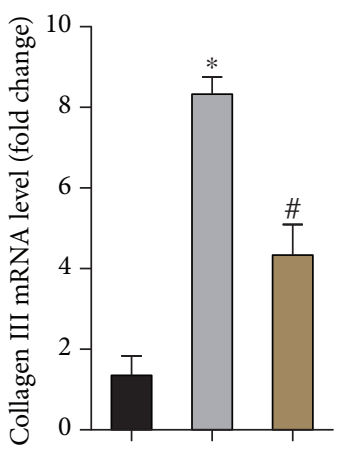

- Normal

- $\mathrm{HG}$

- HG + Ad-SOCS3

(f)

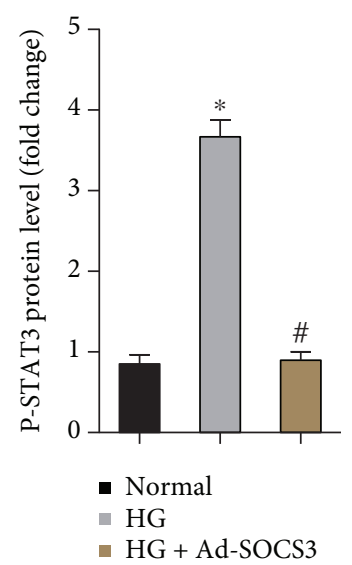

(g) 


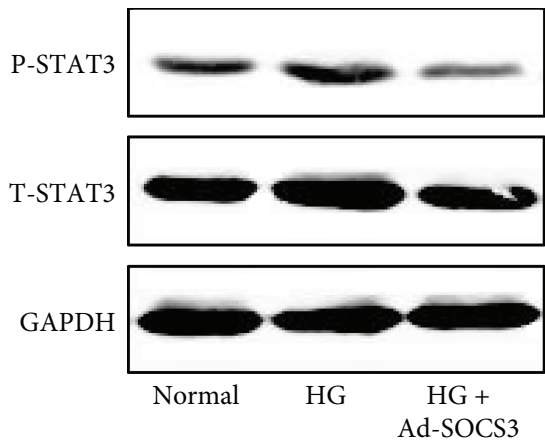

(h)

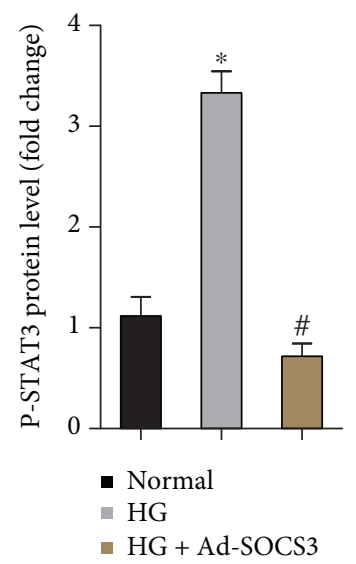

FIGURE 7: The expression and effects of SOCS3 in cardiomyocytes and cardiac fibroblasts. (a) The mRNA level of SOCS3 in cardiomyocytes induced by HG. (b) The mRNA level of SOCS3 in cardiac fibroblasts induced by HG. (c) The immunofluorescence staining of $\alpha$-actin in cardiomyocytes induced by HG with or without SOCS3 overexpression. (d) The immunofluorescence staining of $\alpha$-SMA in cardiac fibroblasts induced by HG with or without SOCS3 overexpression. (e) The mRNA levels of ANP and BNP in cardiomyocytes induced by HG with or without SOCS3 overexpression. (f) The mRNA levels of collagen I and collagen III in cardiac fibroblasts induced by HG with or without SOCS3 overexpression. $(\mathrm{g}, \mathrm{h})$ The protein expression of P-STAT3 and T-STAT3 in the indicated groups. ${ }^{*} P<0.05$ versus normal group and ${ }^{\#} P<0.05$ versus HG group.

least, many of the hub genes and top 5 downregulated or upregulated genes were also implicated with inflammation and immune response: SERPINA3 (immune response to elevated platelet cytosolic $\mathrm{Ca}^{2+}$ ), S100A8 (regulating inflammation and oxidative stress, activatingTLR4 signaling), ANKRD2 (a modulator of NF- $\kappa$ B-mediated inflammatory), FPR1 (G protein-coupled receptor, inflammation), C3 (immune response), CDKN1A (inflammatory response gene), SOCS3 (regulating interleukin), and IL6 (proinflammatory cytokine).

Taken together, our results, in the perspective of bioinformatics, provide compelling evidence for the fact that inflammation and immune effects play critical roles in the development in DCM. Sincerely, we hope that these findings could provide new strategies and insights to identify the pivotal targets and pathways with regards to the immunologic mechanisms of DCM in future research.

4.3. The Improvement of Cardiac Metabolism and Calcium Homeostasis May Benefit DCM. Cardiac metabolic abnormalities are broadly recognized to increase various death risks. In diabetic heart, glucose oxidation was significantly decreased while the intake of fatty acids and its oxidation rate were further elevated. Increased dependence on fatty acids to generate energy may predispose the diabetic heart to the endoplasmic reticulum, oxidative stress, stress, and ischemic damage. Accumulation of intracellular toxic lipid metabolites gives rise to a great many cellular abnormalities resulting in cardiac dysfunction and cardiac remodeling [47]. Meanwhile, the abnormal mitochondrial membrane potential and permeability not only increased the production of reactive oxygen species but also impaired its elimination, causing accumulation of reactive oxygen species in diabetic heart. Excessive production of reactive oxygen species and loss of endothelial antioxidant barrier scales could lead to the production of oxidative stress. Additionally, calcium ion could enter the mitochondrial matrix mediated by a calcium uniporter complex in a sodium calcium exchanger manner, which helps to alleviate calcium overload. In our study, the GO analysis demonstrated that the DEGs were primarily enriched in fatty acid metabolism, mitochondrial membrane potential, mitochondrial uncoupling, glucose transporter, calcium ion binding, and lipid intake.

4.4. SOCS3 May Act as a Novel Therapeutic Target in DCM. The role of the IL-6/STAT3/SOCS3 signaling pathway in tumors has been well evaluated. IL6 stimulates survival, proliferation, and progression to cancer of intestinal epithelial cells via activation of signal transducers and activators of transcription 3 (STAT3), eventually inducing the expression of SCOS3 [47]. Meanwhile, SOCS3 is an important inhibitory factor of STAT3, which could block the phosphorylation of STAT3 and negatively regulate IL-6/STAT3 signaling [25]. Under normal circumstances, the activation of STAT3 is transitory and speedy, while STAT3 could be constitutively activated under pathologic status, which is attributed to the absence or downregulation of SOCS3 [48]. Currently, the role of SCOS3 in DCM remains unclear. We found that both IL6 and SOCS3 acted as hub genes in our study. Therefore, we put forward a hypothesis that IL6 trans-signaling may also activate STAT3/SOCS3, thus promoting the development of DCM. As expected, Western blotting showed that the protein expression of SOCS3 was significantly lower in the DCM group than that in the control group while the phosphorylation of STAT3 was significantly higher in the DCM group, indicating the inhibitory effects of SOCS3 on the phosphorylation of STAT3. To obtain more accurate results, further studies should be performed to explore the 
accurate mechanisms of the IL-6/STAT3/SOCS3 signaling pathway in DCM.

\section{Conclusion}

Conclusively, using a series of bioinformatics analysis, we give a comprehensive and novel illustration of gene expression profiles to identify DEGs expressing in myocardial tissue, which may play critical roles in the occurrence and development in patients with DCM. Genes and pathways implicated with inflammation, immune, and metabolism were significantly altered in DCM. Notably, IL6 may act as a much more important role in the development of DCM beyond our expectation. Additionally, targeting the IL-6/STAT3/SOCS3 signaling pathway is a promising strategy to treat DCM. These findings will greatly contribute to unveiling the molecule mechanisms of DCM. To allow these biomakers and targets to be used more routinely in clinic, further investigations into the correlation of plasma proteins as well as metabolites and these dysregulated genes should be performed.

\section{Data Availability}

The data used to support the findings of this study are available from the corresponding author upon request.

\section{Conflicts of Interest}

No conflict of interest exists.

\section{Authors' Contributions}

Ning Li and Haiming Wu, and Rongxin Geng contributed equally to this work.

\section{Acknowledgments}

This work was supported by grants from the National Natural Science Foundation of China (No. 81470516) and the Key Project of the National Natural Science Foundation (No. 81530012).

\section{Supplementary Materials}

Supplementary 1. Supplemental Table S1: gene-specific primers used in quantitative real-time PCR.

Supplementary 2. Figure S1: the construction of DCM in $\mathrm{db} /$ $\mathrm{db}$ mice. (A) Representative echocardiographic images in the control group and DCM group. (B) Representative images of the H\&E staining and PSR staining in the indicated group.

Supplementary 3. Figure S2: the validation of top 5 upregulated and top 5 downregulated DEGs in vitro. (A) The mRNA expression of NPPA, SFRP4, DSC31, NEB, and FRZB in cardiomyocytes. (B) The mRNA expression of SERPINE1, SERPINA3, ANKRD2, ANKRD2, and S100A8 in cardiomyocytes. (C) The mRNA expression of NPPA, SFRP4, DSC31, NEB, and FRZB in cardiac fibroblasts. (D) The mRNA expression of SERPINE1, SERPINA3, ANKRD2, ANKRD2, and S100A8 in cardiac fibroblasts. ${ }^{*} P<0.05$ versus normal group.

\section{References}

[1] A. Lorenzo-Almorós, J. Tuñón, M. Orejas, M. Cortés, J. Egido, and Ó. Lorenzo, "Diagnostic approaches for diabetic cardiomyopathy," Cardiovascular Diabetology, vol. 16, no. 1, p. 28, 2017.

[2] G. Jia, A. Whaley-Connell, and J. R. Sowers, "Diabetic cardiomyopathy: a hyperglycaemia- and insulin-resistance-induced heart disease," Diabetologia, vol. 61, no. 1, pp. 21-28, 2018.

[3] N. Ghosh and R. Katare, "Molecular mechanism of diabetic cardiomyopathy and modulation of microRNA function by synthetic oligonucleotides," Cardiovascular Diabetology, vol. 17, no. 1, p. 43, 2018.

[4] M. Tate, D. J. Grieve, and R. H. Ritchie, "Are targeted therapies for diabetic cardiomyopathy on the horizon?," Clinical Science, vol. 131, no. 10, pp. 897-915, 2017.

[5] S. Itoh, B. Ding, T. Shishido et al., "Role of p90 ribosomal S6 kinase-mediated prorenin-converting enzyme in ischemic and diabetic myocardium," Circulation, vol. 113, no. 14, pp. 1787-1798, 2006.

[6] D. M. Ouwens, M. Diamant, M. Fodor et al., "Cardiac contractile dysfunction in insulin-resistant rats fed a high-fat diet is associated with elevated CD36-mediated fatty acid uptake and esterification," Diabetologia, vol. 50, no. 9, pp. 19381948, 2007.

[7] B. N. Finck, X. Han, M. Courtois et al., "A critical role for $\operatorname{PPAR} \alpha$-mediated lipotoxicity in the pathogenesis of diabetic cardiomyopathy: modulation by dietary fat content," Proceedings of the National Academy of Sciences of the United States of America, vol. 100, no. 3, pp. 1226-1231, 2003.

[8] Z. Li, C. S. Abdullah, and Z. Q. Jin, "Inhibition of PKC- $\theta$ preserves cardiac function and reduces fibrosis in streptozotocininduced diabetic cardiomyopathy," British Journal of Pharmacology, vol. 171, no. 11, pp. 2913-2924, 2014.

[9] L. Zhang, Y. Yang, L. Cheng, Y. Cheng, H. H. Zhou, and Z. R. Tan, "Identification of common genes refers to colorectal carcinogenesis with paired cancer and noncancer samples," Disease Markers, vol. 2018, Article ID 3452739, 7 pages, 2018.

[10] N. Li, L. Li, and Y. Chen, "The identification of core gene expression signature in hepatocellular carcinoma," Oxidative Medicine and Cellular Longevity, vol. 2018, Article ID 3478305, 15 pages, 2018.

[11] J. Hur, K. A. Sullivan, M. Pande et al., "The identification of gene expression profiles associated with progression of human diabetic neuropathy," Brain, vol. 134, no. 11, pp. 3222-3235, 2011.

[12] Y. Li, Q. Jiang, Z. Ding et al., "Identification of a common different gene expression signature in ischemic cardiomyopathy," Genes, vol. 9, no. 1, p. 56, 2018.

[13] L. Li, Q. Lei, S. Zhang, L. Kong, and B. Qin, "Screening and identification of key biomarkers in hepatocellular carcinoma: evidence from bioinformatic analysis," Oncology Reports, vol. 38, no. 5, pp. 2607-2618, 2017.

[14] R.-X. Geng, N. Li, Y. Xu et al., "Identification of core biomarkers associated with outcome in glioma: evidence from bioinformatics analysis," Disease Markers, vol. 2018, Article ID 3215958, 16 pages, 2018.

[15] P. Gaudet, N. Škunca, J. C. Hu, and C. Dessimoz, "Primer on the gene ontology," in The Gene Ontology Handbook, vol. 1446 of Methods in Molecular Biology, pp. 25-37, Humana Press, New York, NY, USA, 2017. 
[16] M. Kanehisa, "The KEGG database," in 'In Silico' Simulation of Biological Processes: Novartis Foundation Symposium 247, pp. 91-103, John Wiley \& Sons, Ltd., Chichester, UK, 2002.

[17] D. Huang, B. T. Sherman, Q. Tan et al., "The DAVID Gene Functional Classification Tool: a novel biological modulecentric algorithm to functionally analyze large gene lists," Genome Biology, vol. 8, no. 9, p. R183, 2007.

[18] Z. Xu, S. Wang, H. Ji et al., "Broccoli sprout extract prevents diabetic cardiomyopathy via Nrf2 activation in $d b / d b$ T2DM mice," Scientific Reports, vol. 6, no. 1, article 30252, 2016.

[19] N. Zhang, Z. Yang, S. Z. Xiang et al., "Nobiletin attenuates cardiac dysfunction, oxidative stress, and inflammatory in streptozotocin: induced diabetic cardiomyopathy," Molecular and Cellular Biochemistry, vol. 417, no. 1-2, pp. 87-96, 2016.

[20] Z. G. Ma, Y. P. Yuan, X. Zhang, S. C. Xu, S. S. Wang, and Q. Z. Tang, "Piperine attenuates pathological cardiac fibrosis via PPAR- $\gamma$ /AKT pathways," EBioMedicine, vol. 18, pp. 179$187,2017$.

[21] G. Jia, V. G. Demarco, and J. R. Sowers, "Insulin resistance and hyperinsulinaemia in diabetic cardiomyopathy," Nature Reviews Endocrinology, vol. 12, no. 3, pp. 144-153, 2016.

[22] Y. Takeishi and A. Yoshihisa, "Distinct roles of myocardial biopsy in patients with suspected cardiomyopathy," Journal of Cardiac Failure, vol. 22, no. 9, p. S154, 2016.

[23] T. Yoshida, H. Kawano, S. Kusumoto et al., "Relationships between clinical characteristics and decreased plakoglobin and connexin 43 expressions in myocardial biopsies from patients with arrhythmogenic right ventricular cardiomyopathy," International Heart Journal, vol. 56, no. 6, pp. 626-631, 2015.

[24] J. A. Dickerson, S. V. Raman, P. M. Baker, and C. V. Leier, "Relationship of cardiac magnetic resonance imaging and myocardial biopsy in the evaluation of nonischemic cardiomyopathy," Congestive Heart Failure, vol. 19, no. 1, pp. 29-38, 2013.

[25] B. A. Croker, D. L. Krebs, J. G. Zhang et al., "SOCS3 negatively regulates IL-6 signaling in vivo," Nature Immunology, vol. 4, no. 6, pp. 540-545, 2003.

[26] Z. Xing, J. Gauldie, G. Cox et al., "IL-6 is an antiinflammatory cytokine required for controlling local or systemic acute inflammatory responses," The Journal of Clinical Investigation, vol. 101, no. 2, pp. 311-320, 1998.

[27] D. Schmidt-Arras and S. Rose-John, "IL-6 pathway in the liver: from physiopathology to therapy," Journal of Hepatology, vol. 64, no. 6, pp. 1403-1415, 2016.

[28] S. Sarkar, E. Vellaichamy, D. Young, and S. Sen, "Influence of cytokines and growth factors in ANG II-mediated collagen upregulation by fibroblasts in rats: role of myocytes," American Journal of Physiology-Heart and Circulatory Physiology, vol. 287, no. 1, pp. H107-H117, 2004.

[29] G. C. Meléndez, J. L. McLarty, S. P. Levick, Y. du, J. S. Janicki, and G. L. Brower, "Interleukin 6 mediates myocardial fibrosis, concentric hypertrophy, and diastolic dysfunction in rats," Hypertension, vol. 56, no. 2, pp. 225-231, 2010.

[30] Y. Zhang, J. H. Wang, Y. Y. Zhang et al., "Deletion of interleukin-6 alleviated interstitial fibrosis in streptozotocininduced diabetic cardiomyopathy of mice through affecting TGF $\beta 1$ and miR-29 pathways," Scientific Reports, vol. 6, no. 1, article 23010, 2016.

[31] E. Haugen, L. M. Gan, A. Isic, T. Skommevik, and M. Fu, "Increased interleukin-6 but not tumour necrosis factor- alpha predicts mortality in the population of elderly heart failure patients," Experimental \& Clinical Cardiology, vol. 13, no. 1, pp. 19-24, 2008.

[32] F. Papousek, R. Bohuslavova, F. Kolar et al., "Echocardiographic evaluation of Hif- $1 \alpha$ knock-out mice in the early stage of diabetic cardiomyopathy," The FASEB Journal, vol. 27, 2013.

[33] X. Gao, Y. Li, H. Wang, C. Li, and J. Ding, "Inhibition of HIF$1 \alpha$ decreases expression of pro-inflammatory IL- 6 and TNF- $\alpha$ in diabetic retinopathy," Acta Ophthalmologica, vol. 95, no. 8, pp. e746-e750, 2017.

[34] S. Heymans, E. Hirsch, S. D. Anker et al., "Inflammation as a therapeutic target in heart failure? A scientific statement from the Translational Research Committee of the Heart Failure Association of the European Society of Cardiology," European Journal of Heart Failure, vol. 11, no. 2, pp. 119-129, 2009.

[35] K. Savvatis, D. Westermann, H. P. Schultheiss, and C. Tschöpe, "Kinins in cardiac inflammation and regeneration: insights from ischemic and diabetic cardiomyopathy," Neuropeptides, vol. 44, no. 2, pp. 119-125, 2010.

[36] O. Lorenzo, B. Picatoste, S. Ares-Carrasco, E. Ramírez, J. Egido, and J. Tuñón, "Potential role of nuclear factor $\kappa \mathrm{B}$ in diabetic cardiomyopathy," Mediators of Inflammation, vol. 2011, Article ID 652097, 9 pages, 2011.

[37] H. Kaneto, Y. Nakatani, D. Kawamori, T. Miyatsuka, and T. A. Matsuoka, "Involvement of oxidative stress and the JNK pathway in glucose toxicity," The Review of Diabetic Studies, vol. 1, no. 4, pp. 165-174, 2004.

[38] G. Li, E. J. Barrett, M. O. Barrett, W. Cao, and Z. Liu, “Tumor necrosis factor- $\alpha$ induces insulin resistance in endothelial cells via a p38 mitogen-activated protein kinase-dependent pathway," Endocrinology, vol. 148, no. 7, pp. 3356-3363, 2007.

[39] X. Y. Yu, H. M. Chen, J. L. Liang et al., "Hyperglycemic myocardial damage is mediated by proinflammatory cytokine: macrophage migration inhibitory factor," PLoS One, vol. 6, no. 1, article e16239, 2011.

[40] Y. Mano, T. Anzai, H. Kaneko et al., "Overexpression of human C-reactive protein exacerbates left ventricular remodeling in diabetic cardiomyopathy," Circulation Journal, vol. 75, no. 7, pp. 1717-1727, 2011.

[41] G. Torre-Amione, "Immune activation in chronic heart failure," The American Journal of Cardiology, vol. 95, no. 11, pp. 3-8, 2005.

[42] P. M. Becher, D. Lindner, M. Fröhlich, K. Savvatis, D. Westermann, and C. Tschöpe, "Assessment of cardiac inflammation and remodeling during the development of streptozotocin-induced diabetic cardiomyopathy in vivo: a time course analysis," International Journal of Molecular Medicine, vol. 32, no. 1, pp. 158-164, 2013.

[43] B. Dong, D. Qi, L. Yang et al., "TLR4 regulates cardiac lipid accumulation and diabetic heart disease in the nonobese diabetic mouse model of type 1 diabetes," American Journal of Physiology-Heart and Circulatory Physiology, vol. 303, no. 6, pp. H732-H742, 2012.

[44] M. K. Mohammad, M. Morran, B. Slotterbeck et al., "Dysregulated toll-like receptor expression and signaling in bone marrow-derived macrophages at the onset of diabetes in the non-obese diabetic mouse," International Immunology, vol. 18, no. 7, pp. 1101-1113, 2006.

[45] J. Fuentes-Antrás, A. M. Ioan, J. Tuñón, J. Egido, and Ó. Lorenzo, "Activation of toll-like receptors and 
inflammasome complexes in the diabetic cardiomyopathyassociated inflammation," International Journal of Endocrinology, vol. 2014, Article ID 847827, 10 pages, 2014.

[46] G. Giannattasio, S. Ohta, J. R. Boyce, W. Xing, B. Balestrieri, and J. A. Boyce, "The purinergic G protein-coupled receptor 6 inhibits effector $\mathrm{T}$ cell activation in allergic pulmonary inflammation," The Journal of Immunology, vol. 187, no. 3, pp. 1486-1495, 2011.

[47] A. C. Carpentier, "Abnormal myocardial dietary fatty acid metabolism and diabetic cardiomyopathy," Canadian Journal of Cardiology, vol. 34, no. 5, pp. 605-614, 2018.

[48] C. M. Wunderlich, N. Hövelmeyer, and F. T. Wunderlich, "Mechanisms of chronic JAK-STAT3-SOCS3 signaling in obesity," JAK-STAT, vol. 2, no. 2, article e23878, 2013. 


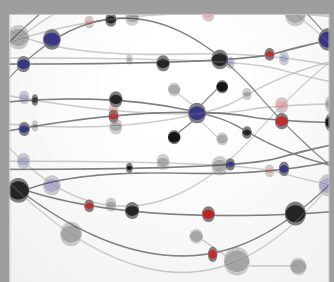

The Scientific World Journal
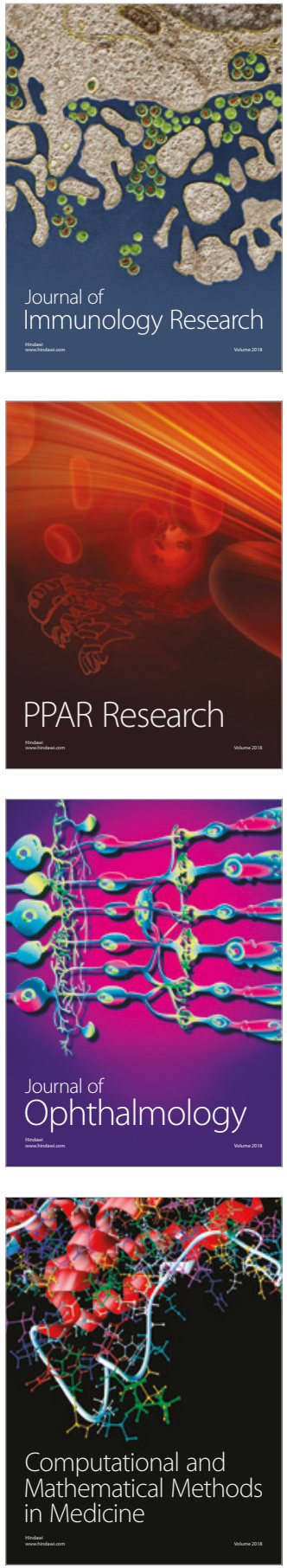

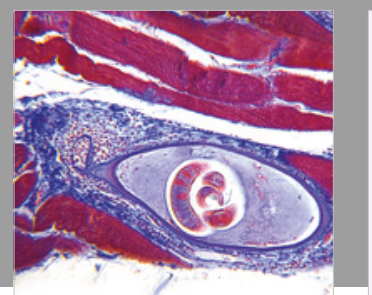

Gastroenterology Research and Practice

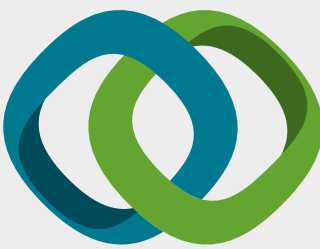

\section{Hindawi}

Submit your manuscripts at

www.hindawi.com
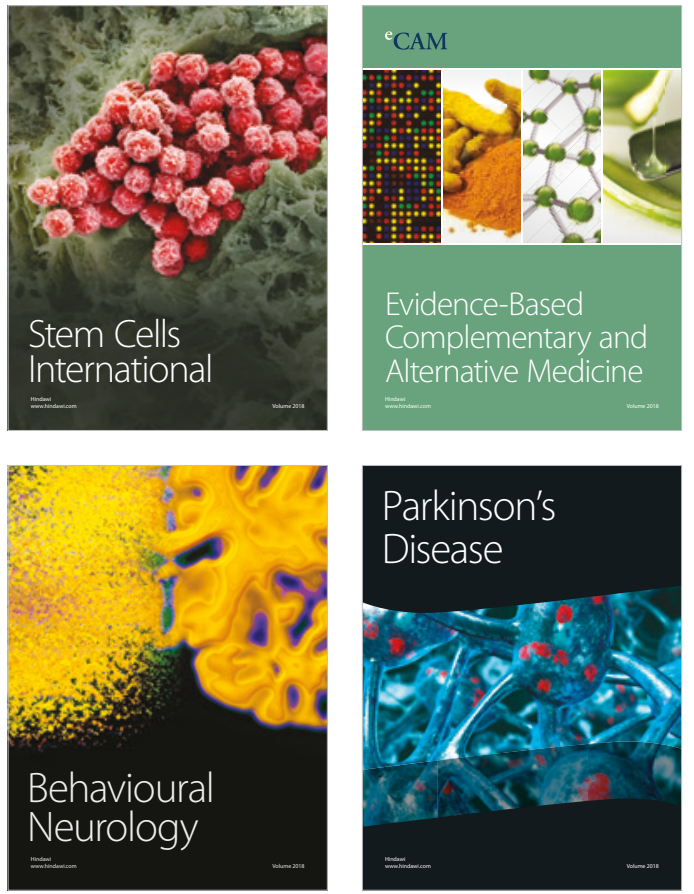

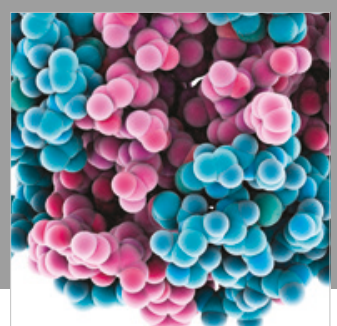

ournal of

Diabetes Research

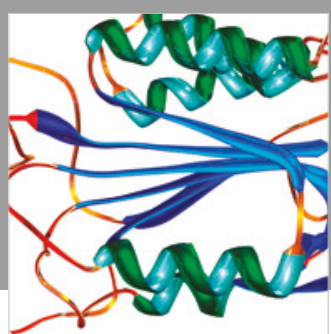

Disease Markers
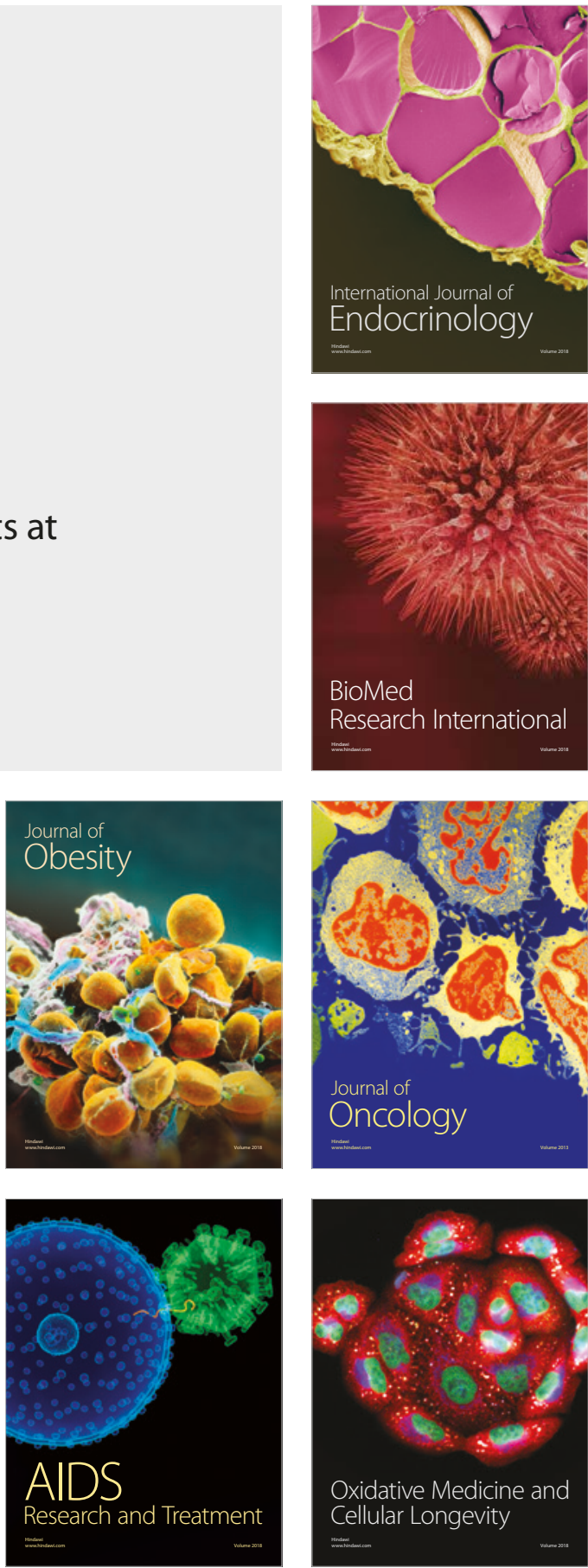